\title{
\begin{tabular}{l|l} 
Mibraries & DSpace@MIT
\end{tabular}
}

\author{
MIT Open Access Articles
}

\section{THE CALIFA AND HIPASS CIRCULAR VELOCITY FUNCTION FOR ALL MORPHOLOGICAL GALAXY TYPES}

The MIT Faculty has made this article openly available. Please share how this access benefits you. Your story matters.

Citation: Bekeraite, S., et al. "THE CALIFA AND HIPASS CIRCULAR VELOCITY FUNCTION FOR ALL MORPHOLOGICAL GALAXY TYPES.” The Astrophysical Journal, vol. 827, no. 2, Aug. 2016, p. L36. (C) 2016 The American Astronomical Society

As Published: http://dx.doi.org/10.3847/2041-8205/827/2/L36

Publisher: American Astronomical Society

Persistent URL: http://hdl.handle.net/1721.1/113891

Version: Final published version: final published article, as it appeared in a journal, conference proceedings, or other formally published context

Terms of Use: Article is made available in accordance with the publisher's policy and may be subject to US copyright law. Please refer to the publisher's site for terms of use. 


\title{
THE CALIFA AND HIPASS CIRCULAR VELOCITY FUNCTION FOR ALL MORPHOLOGICAL GALAXY TYPES
}

\author{
S. Bekeraitė ${ }^{1}$, C. J. Walcher ${ }^{1}$, L. Wisotzki ${ }^{1}$, D. J. Croton ${ }^{2}$, J. Falcón-Barroso ${ }^{3,4}$, M. Lyubenova ${ }^{5}$, D. Obreschkow ${ }^{6}$, \\ S. F. SÁnchez ${ }^{7}$, K. Spekkens ${ }^{8}$, P. Torrey ${ }^{9,10}$, G. van de Ven ${ }^{11}$, M. A. ZWaAn ${ }^{12}$, Y. Ascasibar ${ }^{13}$, J. Bland-Hawthorn ${ }^{14}$, \\ R. González Delgado ${ }^{15}$, B. Husemann ${ }^{16}$, R. A. Marino ${ }^{17,18}$, M. Vogelsberger ${ }^{19}$, And B. Ziegler ${ }^{20}$ \\ ${ }^{1}$ Leibniz-Institut für Astrophysik Potsdam (AIP), An der Sternwarte 16, D-14482 Potsdam, Germany \\ ${ }^{2}$ Centre for Astrophysics and Supercomputing, Swinburne University of Technology, Hawthorn, Victoria 3122, Australia \\ ${ }^{3}$ Dept. Astrofísica, Universidad de La Laguna, C/Astrofísico Francisco Sánchez, E-38205-La Laguna, Tenerife, Spain \\ ${ }^{4}$ Instituto de Astrofísica de Canarias, C/Vía Láctea S/N, E-38200-La Laguna, Tenerife, Spain \\ ${ }^{5}$ Kapteyn Astronomical Institute, University of Groningen, Postbus 800, 9700 AV Groningen, The Netherlands \\ ${ }^{6}$ ICRAR, University of Western Australia, 35 Stirling Highway, Crawley, WA 6009, Australia \\ ${ }^{7}$ Instituto de Astronomía, Universidad Nacional Autonóma de México, A.P. 70-264, 04510, D.F., México \\ ${ }^{8}$ Department of Physics, Royal Military College of Canada, P.O. Box 17000, Station Forces, Kingston, ON, K7K 7B4, Canada \\ ${ }^{9}$ Department of Physics, Kavli Institute for Astrophysics and Space Research, Massachusetts Institute of Technology, Cambridge, MA 02139, USA \\ ${ }^{10}$ TAPIR, Mailcode 350-17, California Institute of Technology, Pasadena, CA 91125, USA \\ ${ }^{11}$ Max Planck Institute for Astronomy, Königstuhl 17, D-69117 Heidelberg, Germany \\ ${ }^{12}$ ESO, ALMA Regional Centre, Karl-Schwarzschild-Strasse 2, D-85748 Garching, Germany \\ ${ }^{13}$ Departamento de Física Teórica, Facultad de Ciencias, Universidad Autónoma de Madrid, E-28049 Madrid, Spain \\ ${ }^{14}$ Sydney Institute for Astronomy, School of Physics, University of Sydney, NSW 2006, Australia \\ ${ }^{15}$ Instituto de Astrofísica de Andalucía (IAA/CSIC), Glorieta de la Astronomía s/n Aptdo. 3004, E-18080 Granada, Spain \\ ${ }^{16}$ European Southern Observatory, Karl-Schwarzschild-Strasse 2, D-85748 Garching b. München, Germany \\ ${ }^{7}$ Department of Physics, Institute for Astronomy, ETH Zürich, CH-8093 Zürich, Switzerland \\ ${ }^{18}$ Departamento de Astrofísica y CC. de la Atmósfera, Facultad de CC. Físicas, Universidad Complutense de Madrid, Avda. Complutense s/n, E-28040 Madrid,Spain \\ ${ }_{19}$ Department of Physics, Kavli Institute for Astrophysics and Space Research, Massachusetts Institute of Technology, Cambridge, MA 02139, USA \\ ${ }^{20}$ University of Vienna, Türkenschanzstr. 17, A-1180 Vienna, Austria \\ Received 2016 May 11; revised 2016 July 22; accepted 2016 August 1; published 2016 August 18
}

\begin{abstract}
The velocity function (VF) is a fundamental observable statistic of the galaxy population that is similar to the luminosity function in importance, but much more difficult to measure. In this work we present the first directly measured circular VF that is representative between $60<v_{\text {circ }}<320 \mathrm{~km} \mathrm{~s}^{-1}$ for galaxies of all morphological types at a given rotation velocity. For the low-mass galaxy population $\left(60<v_{\text {circ }}<170 \mathrm{~km} \mathrm{~s}^{-1}\right)$, we use the HI Parkes All Sky Survey VF. For the massive galaxy population $\left(170<v_{\text {circ }}<320 \mathrm{~km} \mathrm{~s}^{-1}\right)$, we use stellar circular velocities from the Calar Alto Legacy Integral Field Area Survey (CALIFA). In earlier work we obtained the measurements of circular velocity at the $80 \%$ light radius for 226 galaxies and demonstrated that the CALIFA sample can produce volume-corrected galaxy distribution functions. The CALIFA VF includes homogeneous velocity measurements of both late and early-type rotation-supported galaxies and has the crucial advantage of not missing gas-poor massive ellipticals that HI surveys are blind to. We show that both VFs can be combined in a seamless manner, as their ranges of validity overlap. The resulting observed VF is compared to VFs derived from cosmological simulations of the $z=0$ galaxy population. We find that dark-matter-only simulations show a strong mismatch with the observed VF. Hydrodynamic simulations fare better, but still do not fully reproduce observations.
\end{abstract}

Key words: galaxies: evolution - galaxies: kinematics and dynamics - galaxies: statistics

\section{INTRODUCTION}

The circular velocity function (VF), the space density of galaxies as a function of their circular rotation velocities, is directly related to the total dynamical masses of the galaxies and is not dominated by their baryonic content, unlike the galaxy luminosity function (LF; Desai et al. 2004). As a tracer of dark matter halo masses (Zwaan et al. 2010, hereafter Z10), the VF can be used as a test of the $\Lambda$ CDM paradigm (Papastergis et al. 2011; Klypin et al. 2015) and as a probe of cosmological parameters (Newman \& Davis 2000, 2002) or the relation between the dark matter halo and galaxy rotation velocities.

Observationally, VF differs significantly from the LF. The latter, although difficult to predict and interpret theoretically, is much easier to measure (Klypin et al. 2015) and does not depend on the spatial distribution of baryons in galaxies. Depending on the precise definition of circular velocity, the VF is a function of both the halo and baryonic mass spatial distribution and their ratio in a particular galaxy; however, it is not significantly affected by uncertainties in the stellar mass-tolight ratio. In this regard it is a superior tool for testing the results of cosmological simulations.

Measuring the VF is difficult on all halo mass scales. Cluster rotation velocities have completely different dynamical properties and require different observational methods than individual galaxies (Kochanek \& White 2001), while the lowest-velocity galaxy samples are not complete. Even at intermediate velocities the VF has not been fully constrained, because circular velocity measurements for gas-poor earlytypes, dominating the high-velocity end of the galaxy VF, are notoriously challenging (Gonzalez et al. 2000; Papastergis et al. 2011; Obreschkow et al. 2013). Moreover, even though the circular velocity is easy to define theoretically, there is no clear observational definition, especially given that the rotation curves of some classes of galaxies do not flatten. 
Several studies have attempted to use galaxy scaling relations in order to infer circular velocities from more accessible observable quantities. Gonzalez et al. (2000) estimate a VF by converting the SSRS2 LF using the TullyFisher relation. A similar approach was adopted by Abramson et al. (2014), who construct galaxy group and field VFs using velocity estimates based on Sloan Digital Sky Survey (SDSS) photometric data. Desai et al. (2004) construct cluster and field VFs by using SDSS data and Tully-Fisher and Fundamental Plane relations.

In Klypin et al. (2015) a local volume VF, complete down to $v_{\text {circ }} \approx 15 \mathrm{~km} \mathrm{~s}^{-1}$, was estimated using a combination of $\mathrm{HI}$ observations and line-of-sight velocities estimated from photometry. However, their study does not sample the velocities above $\approx 200 \mathrm{~km} \mathrm{~s}^{-1}$.

An HI VF down to $30 \mathrm{~km} \mathrm{~s}^{-1}$ was directly measured from HI Parkes All Sky Survey (HIPASS) line widths (Z10). Nevertheless, as shown in Obreschkow et al. (2013), massive, rapidly rotating, gas-poor ellipticals are systematically missing from HIPASS data. Therefore its high-velocity end is very incomplete.

Papastergis et al. (2011) (P11) estimate the HI line width function from Arecibo Legacy Fast ALFA (ALFALFA) survey data and suggest using the line width function as a more useful probe of the halo mass distribusion. They also provide a VF for all types by combining their VF with the VF converted from Chae (2010) velocity dispersion measurements.

The Calar Alto Legacy Integral Field Area Survey (CALIFA) is in a unique position, due to its well-understood selection function, a wide field of view, and the first IFS sample that includes a large number of galaxies with diverse morphologies. As described in Krajnović et al. (2008), 80\% of early-type galaxies can be expected to have a rotating component. The use of stellar kinematics enables us to include gas-poor, rotating early-type galaxies in a homogeneous manner. Therefore we are able to directly measure the VF for rotating galaxies of all morphological types, in contrast to the inferred VFs reported by Gonzalez et al. (2000), Desai et al. (2004), Chae (2010), and Abramson et al. (2014).

Within this work, we assume a benchmark cosmological model with $H_{0}=70 \mathrm{~km} \mathrm{~s}^{-1} / \mathrm{Mpc}, \Omega_{\Lambda}=0.7$, and $\Omega_{\mathrm{M}}=0.3$. All VFs from the literature were rescaled to this particular cosmology, as described in Croton (2013).

\section{CALIFA STELLAR CIRCULAR VELOCITY MEASUREMENTS}

CALIFA observations use the PMAS instrument (Roth et al. 2005) in PPaK (Verheijen et al. 2004) mode, mounted on the $3.5 \mathrm{~m}$ telescope at the Calar Alto observatory. The CALIFA survey, sample, and data analysis pipeline are described in detail in Sánchez et al. (2012), Husemann et al. (2013), GarcíaBenito et al. (2015), and Walcher et al. (2014). We refer the reader to the first paper of the CALIFA stellar kinematics series (Falcón-Barroso et al., submitted), where the kinematic map extraction and sample are described in full detail.

In this analysis, we use the "useful" galaxy sample defined in Bekeraite et al. (2016, B16) and the circular velocity measurements obtained therein. Briefly, we start with the initial statistically complete sample of 277 available stellar velocity fields, 51 of which were not useful for further analysis due to signal-to-noise ratio issues (low number of Voronoi bins, foreground contamination) or extremely distorted velocity fields. The final sample consisted of 226 galaxies. As shown in B16, the rejected galaxies were predominantly fainter (SDSS $M_{r}>-20 \mathrm{mag}$ ), which affected the lower completeness limit but did not introduce bias in the sample above it.

We then fit the position and rotation curve parameters by performing Markov Chain Monte Carlo (MCMC) modeling of the velocity fields. The rotation velocity $v_{\mathrm{opt}}$ was estimated by evaluating the model rotation velocity at the $80 \%$ light radius (the optical radius). Due to CALIFA's large but still limited field of view, rotation curve extrapolation was necessary for 165 galaxies.

We do not split the galaxy sample into ellipticals and spirals to estimate their $v_{\text {circ }}$ values separately. Instead, as described in Section 4.4 of B16, a correction estimated in Kalinova et al. (2016) has been applied to all galaxies. Kalinova et al. (2016) analyzed the relationship between dynamical masses inferred using the classical ADC approach (see Chapter 4, Binney \& Tremaine 2008) and axisymmetric Jeans anisotropic MultiGaussian models applied to stellar mean velocity and velocity dispersion fields of 18 late-type galaxies observed with the SAURON IFS instrument. We utilize the relation provided in their Table 4 and calculate the circular velocities by multiplying the measured velocity by the square root of the factors provided, based on the ratio between the $v_{\text {opt }}$ and the line-ofsight stellar velocity dispersion at the optical radius. We demonstrate that the obtained circular velocity is comparable with the ionized gas rotation velocity in B16.

\section{RESULTS}

\subsection{CALIFA Circular VF}

We measure the CALIFA stellar circular VF $\Psi_{\text {circ }}$ in the same manner as the LFs in Walcher et al. (2014, W14) and B16, estimating the optimal number of velocity bins using Scott's Rule (Scott 1979). The $1 / V_{\max }$ weights, corrected for cosmic variance as described in W14, are assigned to each galaxy and then used to calculate the VF. We note that the uncertainties correspond to Poissonian errors in each bin only and do not include any uncertainties in circular velocity measurements; see Section 3.2.

As can be seen in Figure 1, the high-velocity end of $\Psi_{\text {circ }}$ lies significantly above the Z10 or P11 HI VFs. This was to be expected since HI surveys are blind to gas-poor massive ellipticals (Obreschkow et al. 2013). However, the CALIFA VF is below the higher-velocity end of the P11 inferred VF for all galaxy types. This is not surprising given that their VF combines the observed ALFALFA VF and the velocity dispersion function of Chae (2010). Our circular VF is defined for rotation-dominated galaxies only and we do not include the velocity dispersion contribution in any way, barring the circular velocity correction described in Section 2.

At lower velocities the CALIFA VF starts to fall off rapidly and deviates from the Schechter function shape. We estimate the region where incompleteness should become important based on the LF of the sample provided in B16. We convert the luminosity completeness limits to velocity using the TullyFisher relation measured in B16 and find that the CALIFA VF should be complete within the velocity range of $140<v_{\text {circ }}<$ $345 \mathrm{~km} \mathrm{~s}^{-1}$. Such a direct conversion excludes the scatter in TFR, which causes a fall-off sooner than would be naively expected from the TFR alone. By taking the rms TFR scatter $(0.27 \mathrm{mag})$ into account, we find that the CALIFA VF, as 

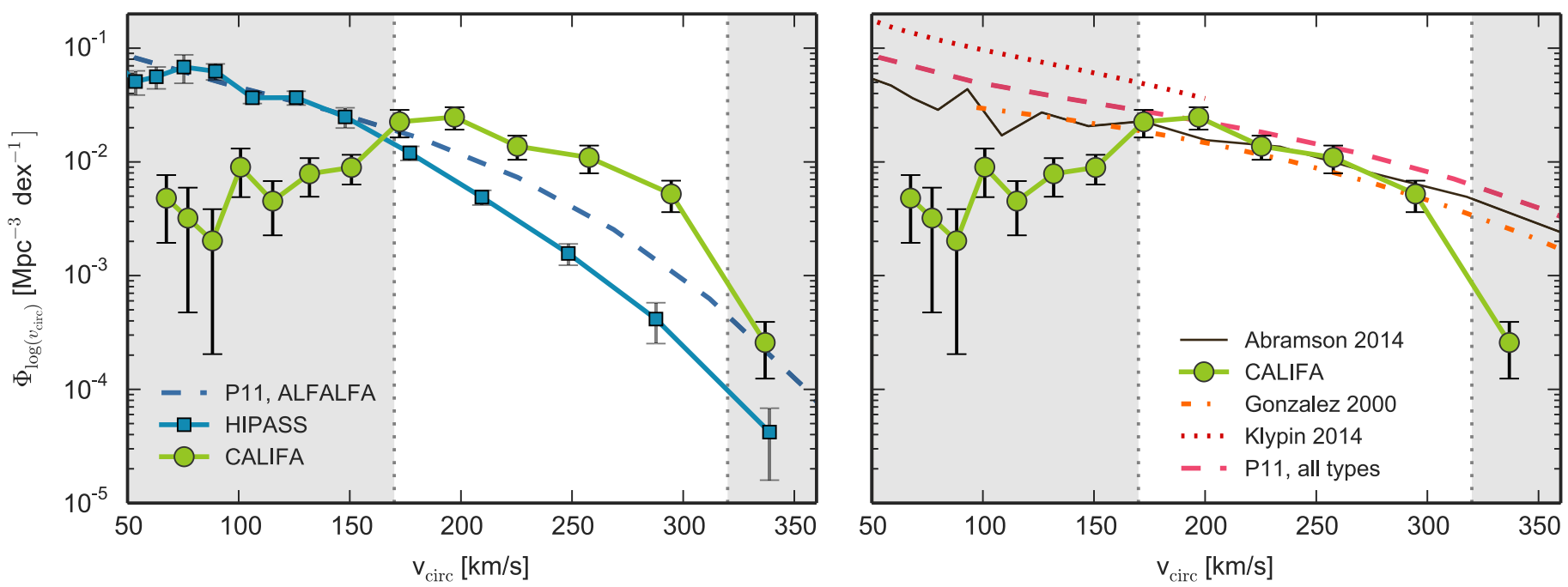

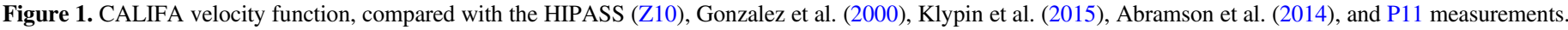

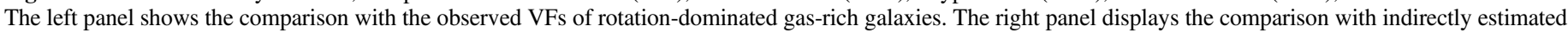
VFs. The shaded areas and dotted vertical lines show an approximate region where incompleteness in the CALIFA sample becomes important.

shown in Figure 1, can be safely assumed to be complete above $170 \mathrm{~km} \mathrm{~s}^{-1}$. At the high-velocity end, the CALIFA survey is limited by its survey volume, as the total number of galaxies brighter than $M_{r}=-23$ expected within the survey is of the order of unity. Given the low number of galaxies at the highvelocity end of the TFR, we are unable to estimate the real TFR scatter among the most massive galaxies and the subsequent onset of bias. However, conservatively adopting the rms TFR scatter of 0.27 mag we find that the CALIFA VF is complete at least up to $320 \mathrm{~km} \mathrm{~s}^{-1}$.

\subsection{Uncertainties}

The CALIFA stellar rotation velocity measurements have significant uncertainties, resulting from a limited spatial resolution of binned stellar velocity fields, a limited CALIFA field of view, and a pressure-support-dependent correction term. The circular VF is likely affected by all these factors. A broader discussion of uncertainties in the circular velocity measurements and volume correction weights is contained in B16 and W14.

In order to check the impact of velocity measurement uncertainties we employ a resampling method similar to P11. We generate 200 mock CALIFA VF samples (shown in Figure 2) in which the volume weights are not changed, but the velocities $v_{\text {circ }}$ are replaced with randomly drawn values such that $v_{\text {circ }}^{\text {test }}=v_{\text {circ }}+\mathcal{N}\left(0, \sigma_{v}\right)$, where $\sigma_{v}$ are the individual velocity uncertainties of each point.

Overall, the effect is a smoothing of the VF as the datapoints are "smeared" into the neighboring bins. As the $1 / V_{\max }$ weights are higher at the lower velocities, this leads to an artificial boost at the highest-velocity end. Undoubtedly, this effect should be present in our VF as well, making the location of the highestvelocity CALIFA datapoint even more uncertain. Given that this bin only includes 3 galaxies and is outside our estimated completeness range, we exclude it from all further analysis.

\subsection{Combined CALIFA-HIPASS Circular Velocity Function}

In order to extend the VF to a wider velocity range we merge the HIPASS VF between 60 and $160 \mathrm{~km} \mathrm{~s}^{-1}$ and CALIFA

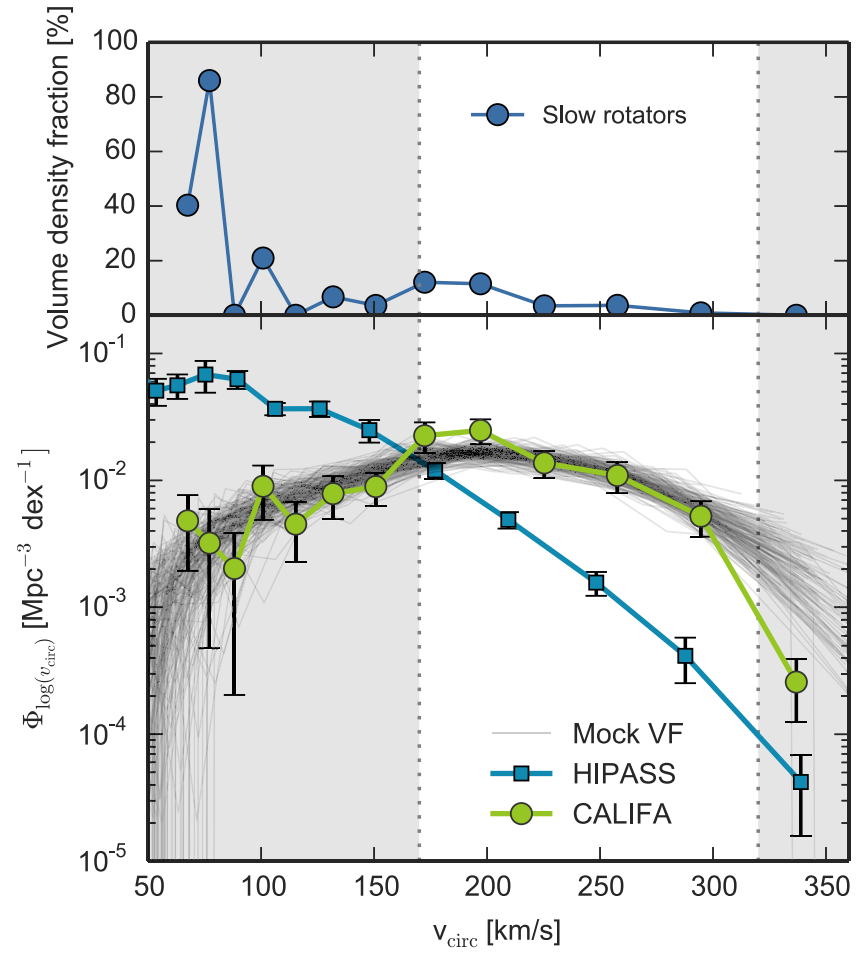

Figure 2. Top panel: the volume density fraction of slow rotators (SR), for which the measured rotation velocities and circular velocity corrections are the most uncertain. This fraction does not reach $20 \%$ of the volume density for $v_{\text {circ }}$ $\gtrsim 110 \mathrm{~km} \mathrm{~s}^{-1}$. Bottom panel: the effect of velocity measurement uncertainties on the velocity function. The thin gray lines are the mock realizations of the VF. The green points and the green line are the CALIFA VF. The blue line shows the HIPASS VF.

circular velocities between 160 and $320 \mathrm{~km} \mathrm{~s}^{-1}$, effectively choosing the more complete VF in each bin. Merging the two VFs in this way is justified as HI-rich late-type galaxies dominate the counts below $200 \mathrm{~km} \mathrm{~s}^{-1}$. At the high-mass limit, early-type massive rotators contribute significantly to the highvelocity end, where the CALIFA sample is expected to be complete at least up to $v_{\text {circ }}=320 \mathrm{~km} \mathrm{~s}^{-1}$, as described above. 


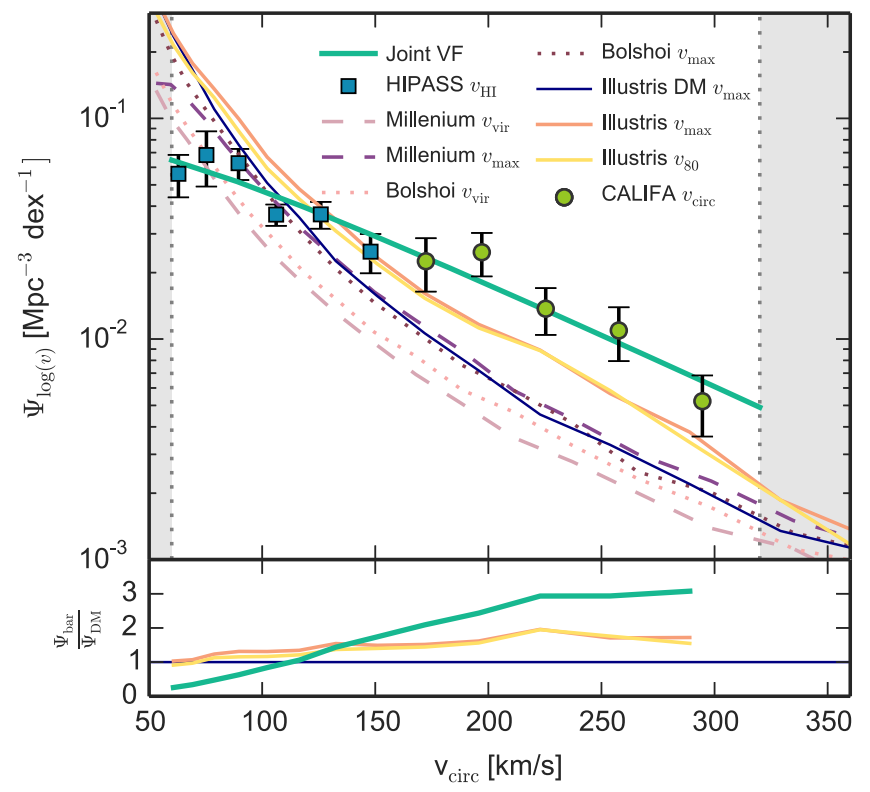

Figure 3. Zoomed-in view showing the combined CALIFA+HIPASS VF and the best Schechter fit, shown as a thick solid teal line. In the top panel, darkmatter-only halo VFs from Millenium and Bolshoi simulations are shown as purple and pink dashed and dotted lines, respectively. $v_{\max }$ VF from Illustris-1 dark-matter-only simulation is shown by a thin dark blue line. Full-physics Illustris-1 simulation VFs, calculated using the subhalo $v_{\max }$ and circular velocity at $80 \%$ stellar mass radius $\left(v_{80}\right)$ are displayed as orange and yellow solid lines, respectively. The lower panel shows the ratio between the baryonic simulated VFs, the combined CALIFA+HIPASS fit and the DM-only Illustris VF. See Section 3.5 for a description, discussion, and references.

We fit a Schechter function

$$
\Psi\left(v_{\text {circ }}\right)=\Psi_{*}\left(\frac{v_{\text {circ }}}{v_{*}}\right)^{\alpha} \exp \left[-\left(\frac{v_{\text {circ }}}{v_{*}}\right)\right],
$$

to the combined VF, shown in Figure 3. The datapoints are listed in Table 2 and the fit parameters are provided in Table 1. Uncertainties for the HIPASS values were taken from Obreschkow et al. (2013), where they supplement direct measurement and shot noise with other uncertainties such as distance errors, cosmology uncertainties, and cosmic variance. Similarly to Z10, we find that the model parameters are highly covariant.

\subsection{Discussion}

Despite the care with which we have undertaken our analysis, combining the HI rotation velocities and stellar circular rotation velocities as we have done has some caveats.

First of all, the actual methods used to construct the HIPASS and CALIFA VFs are different. The CALIFA volume correction procedure uses a more straightforward $1 / V_{\max }$ method (Schmidt 1968) improved by accounting for the radial density variations.

Meanwhile, Z10 employ a bivariate step-wise maximum likelihood (2DSWML) technique to obtain their space densities. Zwaan et al. (2003) verify that the method is insensitive to even large radial density variations. In addition, the HIPASS line width function (WF) matches the WF obtained from the deeper ALFALFA survey down to $60 \mathrm{~km} \mathrm{~s}^{-1}$ (P11), confirming that the effect of large-scale structure on the HIPASS VF is negligible, at least in the range
Table 1

Schechter Function Fit Parameters for CALIFA+HIPASS VF (Equation (1))

\begin{tabular}{lcc}
\hline \hline$\Psi_{*}\left[\times 10^{-3} \mathrm{Mpc}^{-3}\right]$ & $v_{*}\left(\mathrm{~km} \mathrm{~s}^{-1}\right)$ & $\alpha$ \\
\hline $130.0 \pm 35.8$ & $89.3 \pm 32.8$ & $0.2 \pm 0.6$ \\
\hline
\end{tabular}

Table 2

CALIFA-HIPASS Velocity Function Values

\begin{tabular}{lcc}
\hline \hline Survey & $v_{\text {circ }}\left(\mathrm{km} \mathrm{s}^{-1}\right)$ & $\Psi\left(\log _{10} v_{\text {circ }}\right)\left[\times 10^{-3} \mathrm{Mpc}^{-3}\right]$ \\
\hline \multirow{3}{*}{ HIPASS } & 63.0 & $56.1 \pm 12.2$ \\
& 75.3 & $68.2 \pm 19.1$ \\
& 89.5 & $62.7 \pm 10.1$ \\
& 106.2 & $36.6 \pm 4.1$ \\
& 125.9 & $36.7 \pm 5.1$ \\
CALIFA & 147.9 & $24.9 \pm 5.0$ \\
& 172.4 & $22.5 \pm 6.1$ \\
& 197.1 & $24.7 \pm 5.5$ \\
& 225.4 & $13.7 \pm 3.3$ \\
& 257.7 & $10.9 \pm 3.0$ \\
& 294.6 & $5.2 \pm 1.6$ \\
\hline
\end{tabular}

of our analysis. As shown in Zwaan et al. (2003), the $1 / V_{\max }$ and 2DSWML methods yield practically indistinguishable results, confirming that the two VFs derived using both methods are compatible.

The HIPASS sample consists of late-type galaxies only, since visually classified early-type galaxies, comprising $11 \%$ of the sample, have been removed. However, the fraction of earlytype and S0 galaxies is reported to have a noticeable effect on the VF only for galaxies with rotation velocities above $200 \mathrm{~km} \mathrm{~s}^{-1}$, where we use CALIFA VF values already.

HIPASS line widths have been corrected for inclination using SuperCOSMOS imaging $b$-band photometric axis ratios (Meyer et al. 2008), while we use kinematic inclinations obtained from MCMC modeling of the two-dimensional (2D) velocity fields. Photometric inclination estimates are systematically affected by unknown intrinsic disk thickness, choice of $b / a$ measurement radius, and any departure from a perfect circular disk shape. However, given that Z10 exclude galaxies with estimated inclinations $i<45^{\circ}$ due to larger uncertainties at low inclinations, inconsistencies in inclination measurements are unlikely to have had a significant effect.

As discussed in Z10, HIPASS may not detect HI at the flat part of the rotation curve for all galaxies, especially those with $v_{\text {circ }} \leqslant 60 \mathrm{~km} \mathrm{~s}^{-1}$. Similarly, a small fraction of low-mass galaxies might not have enough gas to have been detected by HIPASS. We treat the lowest VF end with caution, and exclude HIPASS datapoints below $60 \mathrm{~km} \mathrm{~s}^{-1}$ from the combined fit. Therefore, the joint VF should be representative in the velocity range of $60<v_{\text {circ }}<320 \mathrm{~km} \mathrm{~s}^{-1}$.

\subsection{Comparison with Simulations}

We compare our work with a number of simulations. Shown in Figure 3 are the VFs from the Millennium (Springel et al. 2005) and Bolshoi (Klypin et al. 2011) dark matter simulations. Here we are plotting friends-of-friends DM halos using two different halo circular velocity definitions: virial velocity $v_{\text {vir }}$ and maximum circular velocity $v_{\max }$. In addition, we show the Illustris-1 DM-only run $v_{\max }$-based VF, constructed for halos with $M_{\mathrm{DM}}>10^{10} M_{\odot}$. 
We also include two VFs measured from Illustris-1 fullphysics simulations (Vogelsberger et al. 2014a, 2014b). Illustris $v_{\max }$ is calculated for all subhalos with stellar masses $M_{*}>10^{8} M_{\odot}$, while Illustris $v_{80}$ is calculated as the gravitational potential-induced circular rotation velocity at the $80 \%$ stellar mass radius.

It is strikingly evident that the observed VF does not agree with the dark-matter-only simulations, even though the lowvelocity end of Bolshoi and Millenium simulations displays marginal agreement with the observational data. At intermediate velocities the dark-matter-only VFs sit well below both the observed data and the baryonic simulation.

However, we find that the observed VF cannot be reconciled with the Illustris $v_{80^{-}}$and $v_{\max }$-based VFs, though the fullphysics simulations produce VFs that are significantly closer to the observed VF. The lack of observed galaxies is evident for velocities lower than $v_{\text {circ }} \approx 120 \mathrm{~km} \mathrm{~s}^{-1}$. This fact was already shown in Gonzalez et al. (2000), Papastergis et al. (2011), Abramson et al. (2014), and Z10, however, we find it worthy to revisit their results using the latest hydrodynamical simulation results.

Interestingly, at the intermediate velocities the predicted VFs are systematically offset from the observations, differing by up to a factor of 3 . This discrepancy is not related to the "underabundance" problem.

The mismatch between simulations and observations is either a result of an inconsistency in the way that observations and simulations are measuring the $\mathrm{VF}$, or the structure of simulated galaxies is inconsistent with the structure of observed galaxies. We have not yet performed a fully fair comparison between the simulations and observations using the $80 \%$ light radius in both cases, employing adequate surface brightness cuts and including projection effects for the simulation. In a very recent paper by Macciò et al. (2016) it was shown that at least some of the tension between the data and models at the low end of the VF can be alleviated by considering the finite extent of $\mathrm{HI}$ disks and relatively larger vertical velocity dispersion. Observational confirmation of their result would go a long way toward explaining the tension in the VF comparison at low circular velocities. We additionally note that while the Illustris VF does not fully match the observed data at high circular velocities, further study of the effects of baryons on the masses and structure of dark matter halos may close the remaining gap. The difference between observed and simulated VFs should be considered to be a constraint on the future generations of galaxy formation models.

\section{CONCLUSIONS}

In this work we measure the CALIFA stellar VF, derived from a sample of 226 stellar velocity fields. To our best knowledge, it is the first directly measured VF that includes early-type fast rotators as well as late-types.

We then combined this VF with the HIPASS VF to obtain the first directly measured VF that simultaneously covers a wide range of circular velocities and morphological types. This has the benefit of using the space density and velocity data measured from the same surveys, without assuming scaling relations or conversions between kinematic observables. The combined VF is complete in the range of $60<v_{\text {circ }}<$ $320 \mathrm{~km} \mathrm{~s}^{-1}$. We find that the Illustris simulation VF does not reproduce the observed data in both the low- and high-velocity ranges.
The differences between $\Lambda \mathrm{CDM}$ predictions and the observed VF are not dissimilar to those found when comparing the halo and stellar mass functions. There, physical processes that are important for galaxy formation cause a decoupling of the halo and galaxy growth. By highlighting similar discrepancies, this work opens a new window for comparison with theory that should deepen our understanding of galaxy evolution. The resulting VF is expected to provide constraints on galaxy assembly and evolution models, and insights into baryonic angular momentum, and is expected to help improve halo occupation distribution and semi-analytic disk formation models.

The authors would like to express gratitude to Louis Abramson for kindly providing VF data and for assistance with interpreting it. We sincerely thank the referee for the insightful comments.

S.B. acknowledges support from BMBF through the Erasmus-F project (grant number 05 A12BA1) and is grateful to Leibniz-Institut für Astrophysik Potsdam for its hospitality during her guest stay there in 2016. J.F.B. acknowledges support from grant AYA2013-48226-C3-1-P from the Spanish Ministry of Economy and Competitiveness (MINECO). C.J.W. acknowledges support through the Marie Curie Career Integration Grant 303912. S.F.S. thanks the CONACYT125180 and DGAPA-IA100815 projects for providing support during this study. D.O. thanks the University of Western Australia for its support via a Research Collaboration Award. K.S. acknowledges funding from the Natural Sciences and Engineering Research Council of Canada.

This study makes use of the data provided by the Calar Alto Legacy Integral Field Area (CALIFA) survey (http://www. califa.caha.es). This work is based on observations collected at the Centro Astronmico Hispano Alemán (CAHA) at Calar Alto, operated jointly by the Max-Planck-Institut fr Astronomie and the Instituto de Astrofisica de Andalucia (CSIC). CALIFA is the first legacy survey being performed at Calar Alto.

We have extensively used the open source data analysis and visualization tools Matplotlib (Hunter 2007) and SciPy (Jones et al. 2001-2015).

\section{REFERENCES}

Abramson, L. E., Williams, R. J., Benson, A. J., Kollmeier, J. A., \& Mulchaey, J. S. 2014, ApJ, 793, 49

Bekeraitè, S., Walcher, J., \& Falcón-Barroso, J. 2016, ApJ, in press

Binney, J., \& Tremaine, S. 2008, Galactic Dynamics (2nd ed.; Princeton, NJ: Princeton Univ. Press)

Chae, K.-H. 2010, MNRAS, 402, 2031

Croton, D. J. 2013, PASA, 30, 52

Desai, V., Dalcanton, J. J., Mayer, L., et al. 2004, MNRAS, 351, 265

García-Benito, R., Zibetti, S., Sánchez, S. F., et al. 2015, A\&A, 576, A135

Gonzalez, A. H., Williams, K. A., Bullock, J. S., Kolatt, T. S., \& Primack, J. R. 2000, ApJ, 528, 145

Hunter, J. D. 2007, CSE, 9, 90

Husemann, B., Jahnke, K., Sánchez, S. F., et al. 2013, A\&A, 549, A87

Jones, E., Oliphant, T., Peterson, P., et al. 2001-2015, SciPy: Open Source Scientific Tools for Python, Online (accessed 2015 June 17)

Kalinova, V., van de Ven, G., Lyubenova, M., et al. 2016, MNRAS, submitted

Klypin, A., Karachentsev, I., Makarov, D., \& Nasonova, O. 2015, MNRAS, 454, 1798

Klypin, A. A., Trujillo-Gomez, S., \& Primack, J. 2011, ApJ, 740, 102

Kochanek, C. S., \& White, M. 2001, ApJ, 559, 531

Krajnović, D., Bacon, R., Cappellari, M., et al. 2008, MNRAS, 390, 93

Macciò, A. V., Udrescu, S. M., Dutton, A. A., et al. 2016, arXiv:1607.01028

Meyer, M. J., Zwaan, M. A., Webster, R. L., Schneider, S., \& Staveley-Smith, L. 2008, MNRAS, 391, 1712 
Newman, J. A., \& Davis, M. 2000, ApJL, 534, L11

Newman, J. A., \& Davis, M. 2002, ApJ, 564, 567

Obreschkow, D., Ma, X., Meyer, M., et al. 2013, ApJ, 766, 137

Papastergis, E., Martin, A. M., Giovanelli, R., \& Haynes, M. P. 2011, ApJ, 739, 38

Roth, M. M., Kelz, A., Fechner, T., et al. 2005, PASP, 117, 620

Sánchez, S. F., Kennicutt, R. C., Gil de Paz, A., et al. 2012, A\&A, 538, A8

Schmidt, M. 1968, ApJ, 151, 393

Scott, D. W. 1979, Biometrika, 66, 605
Springel, V., White, S. D. M., Jenkins, A., et al. 2005, Natur, 435, 629

Verheijen, M. A. W., Bershady, M. A., Andersen, D. R., et al. 2004, AN, 325,151

Vogelsberger, M., Genel, S., Springel, V., et al. 2014a, MNRAS, 444, 1518

Vogelsberger, M., Genel, S., Springel, V., et al. 2014b, Natur, 509, 177

Walcher, C. J., Wisotzki, L., Bekeraité, S., et al. 2014, A\&A, 569, A1

Zwaan, M. A., Meyer, M. J., \& Staveley-Smith, L. 2010, MNRAS, 403, 1969

Zwaan, M. A., Staveley-Smith, L., Koribalski, B. S., et al. 2003, AJ, 125, 2842 